\section{A computer program for interpersonal perception method}

\author{
S. R. VENKATRAMAIAH and \\ K. PARVATHESWARA PRASAD \\ Sri Venkateswara University, Tirupati, 517502, India
}

The computer program reported here facilitates analysis of the interpersonal perception method (IPM), which deals with large numbers of dyads.

Description of IPM. The interpersonal perception method (IPM) was designed and developed by Laing, Phillipson, and Lee (1966) to measure and provide information about conjunctions and disjunctions of two persons on a range of important issues $(\mathrm{X})$ involved in their dyadic interaction. The method deals with experiences, perceptions, and actions that take place when two persons are engaged in a meaningful encounter, such as those involving husband $(\mathrm{H})$ and wife $(\mathrm{W})$, father $(\mathrm{F})$ and son (S), therapist (T) and patient (P).

IPM consists of 60 dyadic issues. Each issue $(X)$ has a set of 12 items. These 12 items are further divided into three subsets, with four items within each subset. Thus, there are 720 items $(60 \times 3 \times 4)$ in all, and each person in the dyad answers every item separately. The average time required to complete 720 items is about $90 \mathrm{~min}$.

Each dyad has two epicenters of experience, two points of view, or two perspectives, e.g., a husband's perspective (H) and a wife's perspective (W). These perspectives have, further, four relationships within the dyadic system: (1) husband's relationship with himself (HH), (2) husband's relationship with wife (HW), (3) wife's relationship with herself (WW), and (4) wife's relationship with husband (WH). Accordingly, there are four items eliciting responses in each phase of the relationships.

In addition, every issue $(\mathrm{X})$ is perceived by the persons in the dyad at three levels of perspectives: direct level, e.g., husband's view of $(\mathrm{X}), \mathrm{H}(\mathrm{X})$; meta level, e.g., husband's view of wife's view of $(\mathrm{X}), \mathrm{HW}(\mathrm{X})$; meta-meta level, e.g., husband's view of wife's view of his view of $(X), \operatorname{HWH}(X)$.

Thus, four directions of relationships and three levels of perspectives in respect to 60 issues account for the 720 items on the IPM.

The method of processing the data, consisting of 1,440 responses $(720 \times 2)$, is complex and time consuming (Laing et al., 1966, Chapter V, p. 49). Two types of analysis have been proposed: nonreciprocally matched

Requests for reprints should be sent to Dr. S. R. Venkatramaiah, Reader in Clinical Psychology, Faculty of Home Science, S. V. University College, Tirupati, 517502, India, or to K. Parvatheswara Prasad, Faculty of Electrical Engineering, S. V. University Engineering College, Tirupati, 517502, India. The authors are grateful to Sri Venkateswara University authorities for granting financial assistance in support of the project. comparisons and reciprocally matched comparisons. The method of reciprocally matched comparisons is more useful for drawing up a profile of the relationship between the persons in the dyad. The analysis requires 1,200 comparisons of responses and the tabulation of results as percent frequencies under categories (see Table 1) for each of the four relationships.

Program Description. There are two subscripted logical quantities, HUS (I, J, M) and WIF (I, K, M), taking only true or false values. The first subscript of the variables refers to the item number; the second subscripts, $\mathrm{J}$ and $\mathrm{K}$, refer to the level along the spiral, $\mathrm{H}_{1}, \mathrm{H}_{2}$, or $\mathrm{H}_{3}$ and $\mathrm{W}_{1}, \mathrm{~W}_{2}$, or $\mathrm{W}_{3}$, respectively, and the third subscript, $M$, refers to the four phases of relationships, HH, HW, WH, or WW, within the dyadic system. There are also two single-subscripted and 12 doublesubscripted integer variables (as listed in Table 1) that are indices or counters corresponding to the various categories. Being counters, they are duly initialized at the beginning of the program.

The various comparisons to be made for finding conjunction or disjunction between any pair of HUS (I, J, M) and WIF (I, K, M) is effected through an inclusive-AND operation (represented by the symbol - ) on the two logical variables. The result of this operation, which is again logical, is true if both HUS (I, J, M) and WIF (I, K, M) are both true or false, and false if either of them is false. The former condition represents a conjunction and the latter a disjunction between the two variables. As this logical operation is performed a number of times in the course of the program, a logical function subprogram, MATCH, defined as MATCH (HUS, WIF, I, J, K, M) = HUS (I, J, M) $\odot$ WIF (I, K, M), is frequently referred to in the main program.

The method of IPM is essentially an exercise in evaluating MATCH for a number of HUS and WIF variables, and counting the number of times MATCH is

\section{Table 1}
1. Agreement (AGR)
2. Disagreement (DISAGR)
3. Understanding (UND)
4. Misunderstanding (MIS)
5. Agreement + Understanding (AU)
6. Disagreement + Understanding (DU)
7. Agreement + Misunderstanding (AM)
8. Disagreement + Misunderstanding (DM)
9. Realization (REALIZ)
10. Failure to Realize (FAILRZ)
11. Realization + Understanding (RU)
12. Failure to Realize + Understanding (FU)
13. Realization + Misunderstanding (RM)
14. Failure to Realize + Misunderstanding (FM)

Note-More redundant combinations of categories are suggested by Laing et al., 1966. 
true and false. The counting is done by defining a number of indices as listed in Table 1. Provision is also made for finding percentage measures of these raw scores. The variables prefixed with the letter "P" corresponding to Table 1 are identified as the percentages of the respective scores.

The logical variables, INDEX, are helpful in the determination of various combinations of Agreement (or otherwise), Understanding (or otherwise), and Realization (or otherwise), e.g., AU, DM, RM, etc. They are set to false at the beginning and at the end of every calculation cycle. Certain other variable names like $\mathbb{I N}, \mathrm{C}, \mathrm{D}$, etc., find a place in the program only to make it more efficient.

Input and Output. The 1,440 logical values of HUS and WIF constitute the data of the program and are supplied as ones or zeros only to be converted to true or false later. All the indices listed in Table 1, and their respective percentage measures, form the output of the program and are printed out to a convenient desired format. The final tabulated scores satisfy the inherent checks available in IPM, like AU $(\mathrm{L}, \mathrm{M})+\mathrm{AM}(\mathrm{L}, \mathrm{M})$ = AGR (M).

Memory and Time Requirements. The problem is coded in FORTRAN IV (Note 1). This program was run on an IBM System 370, Model 155, at the Computer
Center, Indian Institute of Technology, Madras. The source deck contains 156 cards. The problem required a core storage of 16,672 bytes (or approximately $16 \mathrm{~K}$ ) to load the problem. Total time was $3.35 \mathrm{sec}, 1 \mathrm{sec}$ for compilation and $2.35 \mathrm{sec}$ for execution. It is possible to reduce the effective array area required by commoning IN and indices listed in Table 1 and declaring them as half-word integers, though we have not attempted it. Further reduction in core storage can be effected by requiring only 1 byte, instead of one word, for storage of any logical variable by a suitable type declaration.

Availability. Copies of a source listing with a sample input and output can be obtained from either author on request.

\section{REFERENCE NOTE}

IBM System 370 FORTRAN Manual, New York: International Business Machines Corporation, 1970.

\section{REFERENCE}

Laing, R. D., Phillipson, H.. \& Lee, A. R. Interpersonal Perception. London: Tavistock, 1966. 